\title{
The application of the associative mean spherical approximation in the theory of nonaqueous electrolyte solutions
}

\author{
J.Barthel ${ }^{1}$, H.Krienke ${ }^{1}$, M.F.Holovko ${ }^{2}$, V.I.Kapko ${ }^{2}$, \\ I.A.Protsykevich ${ }^{2}$ \\ 1 Institut für Physikalische und Theoretische Chemie, Universitat \\ Regensburg, D-93040, Regensburg, Germany \\ 2 Institute for Condensed Matter Physics \\ of the National Academy of Sciences of Ukraine, \\ 1 Svientsitskii Str., 79011 Lviv, Ukraine
}

Received June 9, 2000

Ionic association in electrolyte solutions is investigated in the framework of chemical models. The associative mean spherical approximation (AMSA) for electrolyte theory is reviewed. It is shown that AMSA in combination with the Ebeling association constant satisfactorily reproduces the thermodynamic measurement data up to high concentrations for nonaqueous solutions of solvents of relative permittivities in the range $20<\varepsilon<36$. For ionic solutions of lower permittivity the AMSA is modified by including ion trimers and tetramers to obtain a correct description of osmotic coefficients and ionic conductivities.

Key words: electrolyte solutions, ionic association, associative mean spherical approximation, osmotic and activity coefficients, ionic conductivity

PACS: $61.20 . \mathrm{Qg}, 66.10 . \mathrm{Ed}$

\section{Introduction}

It is our pleasure to dedicate this article to I.R. Yukhnovskii whose fundamental work in statistical physics yielded a considerable progress in the theory of electrolyte solutions. To his work belongs a general scheme of plasma-parameter expansions for pair distribution functions of ionic fluids [1], the application of the collective variables method to the correct treatment of long and short-range interionic interactions [2] and the development of the ion-molecular approach for the correct description of solvation phenomena in electrolyte solutions [3-6].

Another important aspect of electrolyte solutions is connected with the ionic association concept introduced by Bjerrum [7] and used in the chemical model ap- 
proach $[8,9]$. In the framework of this approach the electrolyte is considered to be a mixture of free ions and ion aggregates (usually ion pairs and sometimes trimers, tetramers etc.) which are assumed to take part in chemical equilibrium according to the corresponding mass action law (MAL). For the description of such mixtures the theory of ionic fluids is traditionally modified by simply correcting the ion concentrations using the concentration of free ions obtained from the MAL [7-9]. However this approach neglects the contributions of electrostatic interaction from ionic aggregates and therefore is correct only in the regime of weak association. At the increasing association, the electrostatic contributions of the ion aggregates increase and are not negligible.

Another route [10] starts from the associative mean spherical approximation (AMSA) [11] based on the theory of associating fluids [12-14]. It was shown that this approach coincides with the traditional approach of weak association, but also is correct in the regime of strong association. With Ebeling's expression of the association constant $[15,16]$ the theory correctly reproduces both the high coupling limit of ion association and the low density limit. This theory reproduces satisfactorily the experimental osmotic coefficients of nonaqueous electrolyte solutions with solvents of relative permittivities in the range $20<\varepsilon<36$ up to high ion concentrations [10]. For solvents with lower permittivities the theory was modified to take into account the effect of ion trimers and tetramers [17], and then satisfactorily reproduces the experimental data of low permittivity electrolyte solutions.

This AMSA approach will be reviewed in the present paper. Applications used for the description of the concentration dependence of vapour pressures and conductivities of nonaqueous solutions of associating electrolytes will be discussed.

\section{Model and theory}

The description of thermodynamic and transport excess properties of nonaqueous electrolyte solutions is based on the ionic approach (McMillan-Mayer level) using a chemical model considering free ions and ion associates in equilibrium. The effect of solvent molecules is accounted for by introducing the permittivity $\varepsilon$ into Coulomb's interaction law and by appropriately choosing the short-range part of interionic interactions.

We begin with the formation of electrically neutral ionic pairs in the framework of the restricted primitive model given by the following interactions

$$
U_{a b}(r)= \begin{cases}\infty, & r<R, \\ \frac{Z_{a} Z_{b} e^{2}}{\varepsilon r}, & r>R,\end{cases}
$$

where $Z_{+}=-Z_{-}=1, R$ is the diameter of ions, $e$ is the elementary charge, $\varepsilon$ is the solvent permittivity.

After solving the corresponding AMSA for this model we will modify the results obtained to take into account the possibilities of trimer and tetramer formation. 


\subsection{AMSA theory}

AMSA theory represents the two-density version of the traditional mean spherical approximation $[18,19]$ for an ionic fluid of associative particles. To treat the ion pairs we introduce in the chemical picture in addition to the charged hard sphere interaction, equation (1), a formal short range cation-anion interaction, $U_{+-}^{\text {as }}(r)$, which is responsible for the formation of electrically neutral ion pairs $\left[C^{+} A^{-}\right]^{0}$ fixed by the value of the second ionic virial coefficient of the system in Ebeling's physical picture of complete dissociation $[15,16]$.

The formation of ion pairs determines the concentration of free ions $c_{+}=c_{-}=\alpha c$ according to the MAL

$$
\frac{1-\alpha}{\alpha^{2}}=c K_{\mathrm{A}} \frac{\left(y_{ \pm}^{\prime}\right)^{2}}{y_{0}^{\prime}},
$$

where $\alpha$ is the degree of dissociation, $c=\rho / 2 N_{\mathrm{A}}$ is the analytical electrolyte concentration, $\rho=\rho_{+}+\rho_{-}$is the total number density, $N_{\mathrm{A}}$ is the Avogadro constant, $K_{\mathrm{A}}$ is the equilibrium constant of ion-pair formation, $y_{ \pm}^{\prime}$ is the mean activity coefficient of the free ions in solution, and $y_{0}^{\prime}$ is that of the ion pairs.

The MAL (2) is written in the form [12-14]

$$
\frac{1-\alpha}{\alpha^{2}}=4 \pi c N_{\mathrm{A}} \int_{0}^{\infty} f_{+-}^{\text {as }}(r) g_{+-}^{00}(r) r^{2} \mathrm{~d} r
$$

where $f_{+-}^{\text {as }}(r)=\exp \left(-\beta U_{+-}^{\text {as }}(r)\right)-1$ is the Mayer function for the associative interaction, $\beta=1 / k T, T$ is the temperature, $k$ is the Boltzmann constant.

At the sticky limit follows

$$
f_{+-}^{\text {as }}(r)=B \delta(r-R)
$$

and the equation (3) can be rewritten in the form

$$
\frac{1-\alpha}{\alpha^{2}}=c 4 \pi N_{\mathrm{A}} B R^{2} g_{+-}^{00}(R)
$$

where $g_{+-}^{00}(R)$ is the contact value at $r=R$ of the pair-distribution function of the unbound oppositely charged ions.

From the comparison of equations (5) and (2) for charged hard spheres follows

$$
K_{\mathrm{A}}=4 \pi N_{\mathrm{A}} B R^{2} \mathrm{e}^{b}, \quad \frac{\left(y_{ \pm}^{\prime}\right)^{2}}{y_{0}^{\prime}}=\mathrm{e}^{-b} g_{+-}^{00}(R)
$$

where $b=e^{2} /(\varepsilon k T R)$ is the Bjerrum parameter characterising the Coulomb interactions between ions at contact distance, and one has [15]

$$
K_{\mathrm{A}}=8 \pi N_{\mathrm{A}} R^{3} \sum_{m \geqslant 2} \frac{b^{2 m}}{(2 m) !(2 m-3)} .
$$


The total pair correlation function $h_{i j}(r)$ between two ions $i$ and $j$ is represented as a sum of four terms $[10-12]$

$$
h_{i j}(r)=h_{i j}^{00}(r)+h_{i j}^{01}(r)+h_{i j}^{10}(r)+h_{i j}^{11}(r),
$$

where the upper index 0 announces that the corresponding ion is free, and the index 1 indicates, when it is bounded in an ion pair, the function $g_{i j}^{00}(r)=h_{i j}^{00}(r)+1$.

The functions $h_{i j}^{\alpha \beta}(r)$ satisfy the system of Wertheim-Ornstein-Zernike (WOZ) equations $[11,12]$

$$
\mathbf{h}_{i j}\left(r_{12}\right)=\mathbf{C}_{i j}\left(r_{12}+\sum_{l} \rho_{l} \int \mathbf{C}_{i l}\left(r_{13}\right) \mathbf{x h}_{l j}\left(r_{32}\right) \mathrm{d} \bar{r}_{3},\right.
$$

where the matrices $\mathbf{h}_{i j}(r)$ and $\mathbf{C}_{i j}(r)$ have the elements $h_{i j}^{\alpha \beta}(r)$ and $C_{i j}^{\alpha \beta}(r)$, and

$$
\mathbf{x}=\left(\begin{array}{ll}
1 & 1 \\
1 & 0
\end{array}\right)
$$

As usual, due to the symmetry of the RPM it is possible to define the sum and the difference functions

$$
h_{\mathrm{s}}^{\alpha \beta}(r)=\frac{1}{2}\left(h_{++}^{\alpha \beta}(r)+h_{+-}^{\alpha \beta}(r)\right), \quad h_{\mathrm{D}}^{\alpha \beta}(r)=\frac{1}{2}\left(h_{++}^{\alpha \beta}(r)-h_{+-}^{\alpha \beta}(r)\right)
$$

and corresponding expressions for the $C$-functions.

Then the WOZ equation (9) decouples into a set of two matrix equations

$$
\begin{aligned}
\mathbf{h}_{\mathrm{s}}\left(r_{12}\right) & =\mathbf{C}_{\mathrm{s}}\left(r_{12}\right)+\rho \int \mathbf{C}_{\mathrm{s}}\left(r_{13}\right) \mathbf{x h}_{\mathrm{s}}\left(r_{32}\right) \mathrm{d} \bar{r}_{3}, \\
\mathbf{h}_{\mathrm{D}}\left(r_{12}\right) & =\mathbf{C}_{\mathrm{D}}\left(r_{12}\right)+\rho \int \mathbf{C}_{\mathrm{D}}\left(r_{13}\right) \mathbf{x h}_{\mathrm{D}}\left(r_{32}\right) \mathrm{d} \bar{r}_{3} .
\end{aligned}
$$

The AMSA closures for the electroneutral sum problem (index $s$ ) are the same as for the associative hard sphere Percus-Yevick (PY) approximation [20]

$$
\begin{array}{r}
h_{\mathrm{s}}^{00}(r)=-1, \quad h_{\mathrm{s}}^{00}(r)=h_{\mathrm{s}}^{10}(r)=h_{\mathrm{s}}^{11}(r)=0, \quad r<R ; \\
C_{\mathrm{s}}^{00}(r)=C_{\mathrm{s}}^{01}(r)=C_{\mathrm{s}}^{10}(r)=0, \quad C_{\mathrm{s}}^{11}(r)=\frac{(1-\alpha)}{4 \pi \rho} \delta(r-R), \quad r \geqslant R .
\end{array}
$$

Similarly, this follows for the difference case (index $D$ )

$$
\begin{array}{r}
h_{D D}^{00}(r)=h_{\mathrm{D}}^{01}(r)=h_{\mathrm{D}}^{10}(r)=h_{\mathrm{D}}^{11}(r)=0, \quad r<R ; \\
C_{\mathrm{D}}^{00}(r)=-\frac{b R}{r}, C_{\mathrm{D}}^{01}(r)=C_{\mathrm{D}}^{10}(r)=0, C_{\mathrm{D}}^{11}(r)=-\frac{(1-\alpha)}{4 \pi \rho} \delta(r-R), \quad r \geqslant R .
\end{array}
$$

The function $g_{+-}^{00}(r)$, introduced into the MAL, equation (5), is defined by

$$
g_{+-}^{00}(R)=1+h_{+-}^{00}(R)=1+h_{\mathrm{s}}^{00}(R)-h_{\mathrm{D}}^{00}(R)
$$


or in its exponential approximation [21]

$$
g_{+-}^{00}(R)=\left[1+h_{\mathrm{s}}^{00}(R)\right] \exp \left[-h_{\mathrm{D}}^{00}(R)\right] .
$$

The solution for $\mathbf{h}_{\mathbf{s}}(r)$ formally coincides with Wertheim's solution [20] for dimerizing hard spheres with $\alpha$ calculated from equation (5). The solution for $\mathbf{h}_{\mathrm{D}}(r)$ was also obtained $[10,11]$ and here we briefly repeat the general features of this solution. Using the Wertheim-Baxter factorization technique, equation (13) is transformed into two equations:

$$
\begin{aligned}
& \mathbf{S}_{\mathrm{D}}(r)=\mathbf{Q}(r)-\int_{0}^{\infty} \mathrm{d} t \mathbf{Q}(r+t) \mathbf{x} \mathbf{Q}^{T}(t), \\
& \mathbf{J}_{\mathrm{D}}(r)=\mathbf{Q}(r)+\int_{0}^{\infty} \mathrm{d} t \mathbf{J}_{\mathrm{D}}(|r-t|) \mathbf{x} \mathbf{Q}(t),
\end{aligned}
$$

where

$$
J_{\mathrm{D}}^{\alpha \beta}(r)=2 \pi \rho \int_{r}^{\infty} s h_{\mathrm{D}}^{\alpha \beta}(s) \mathrm{d} s, \quad S_{\mathrm{D}}^{\alpha \beta}(r)=2 \pi \rho \int_{r}^{\infty} s C_{\mathrm{D}}^{\alpha \beta}(s) \mathrm{d} s .
$$

After differentiation equations (18)-(19) read

$$
\begin{aligned}
2 \pi \rho r \mathbf{C}_{\mathrm{D}}(r) & =-\mathbf{Q}^{\prime}(r)+\int_{0}^{\infty} \mathrm{d} t \mathbf{Q}^{\prime}(r+t) \mathbf{x} \mathbf{Q}^{T}(t) \\
2 \pi \rho r \mathbf{h}_{\mathrm{D}}(r) & =-\mathbf{Q}^{\prime}(r)+2 \pi \rho \int_{0}^{\infty} \mathrm{d} t(r-t) \mathbf{h}_{\mathrm{D}}(|r-t|) \mathbf{x} \mathbf{Q}(t) .
\end{aligned}
$$

From the AMSA closures $\mathbf{Q}(r)$ has the form

$$
\mathbf{Q}(r)=\mathbf{q}(r)-\lim _{\mu \rightarrow 0+} \mathbf{A} \mathrm{e}^{-\mu r},
$$

where

$$
\mathbf{q}(r)=\mathbf{q}_{0}+\mathbf{q}_{1}(r-R), \quad r \leqslant R, \quad \mathbf{q}(r)=0, \quad r>R .
$$

The matrix $\mathbf{A}$ has nonzero values only in the first row

$$
A^{\alpha \beta}=a_{\beta} \delta_{\alpha 0}
$$

Taking into account the structure of $h_{\mathrm{D}}^{\alpha \beta}(r)$ for $r \leqslant R$ according to equation (15) we have from equation (19) for the matrix elements of $\mathbf{q}_{1}$

$$
q_{1}^{\alpha \beta}=J_{\alpha} a_{\beta}
$$

where

$$
J_{\alpha}=2 \pi \rho \int_{0}^{\infty} r \mathrm{~d} r \sum_{\beta} h_{\mathrm{D}}^{\alpha \beta}(r) .
$$

From the boundary conditions one has

$$
q_{0}^{00}=q_{0}^{01}=q_{0}^{10}=0, \quad q_{0}^{11}=-\frac{1-\alpha}{2} .
$$


From equation (21) in the asymptotic cases $r \rightarrow \infty$ and $r \rightarrow 0$ four independent equations are obtained

$$
\begin{aligned}
& (\kappa R)^{2}=a_{0}^{2}+2 a_{0} a_{1} \\
& 2 a_{1}+(\kappa R)^{2} J_{1} R+a_{0}(1-\alpha)=0 \\
& (\kappa R)^{2}\left(1+J_{0} R\right)^{2}+2 a_{0} J_{0} R=0 \\
& a_{0} J_{1} R+a_{1} J_{0} R+\left(1+J_{0} R\right)\left(\kappa^{2} R^{2} J_{1} R+\frac{1}{2} a_{0}(1-\alpha)\right)=0
\end{aligned}
$$

which make up a system of equations for the four parameters $J_{0}, J_{1}, a_{0}$ and $a_{1}$ depending on $\alpha$ and $\kappa^{2} R^{2}$, where $\kappa^{2}=4 \pi e^{2} \beta \rho e^{2} / \varepsilon$ is the Debye-Huckel screening parameter. After some algebra this system can be presented as a single nonlinear equation of the parameter $J_{0}$

$$
(\kappa R)^{2}\left(1+J_{0} R\right)^{4}\left[\alpha-J_{0} R(1-\alpha)\right]=4\left(J_{0} R\right)^{2} .
$$

This equation can be written in another form, if Blum's screening parameter $\Gamma_{B}$ instead of $J_{0}$ is introduced

$$
J_{0} R=-\frac{\Gamma_{\mathrm{B}} R}{1+\Gamma_{\mathrm{B}} R} .
$$

Then a simple nonlinear equation is derived for the scaling parameter $\Gamma_{\mathrm{B}}$ first given by Bernard and Blum [21]

$$
4\left(\Gamma_{\mathrm{B}} R\right)^{2}\left(1+\Gamma_{\mathrm{B}} R\right)^{2}=(\kappa R)^{2} \frac{\alpha+\Gamma_{\mathrm{B}} R}{1+\Gamma_{\mathrm{B}} R} .
$$

The equations (32) and (30) containing the degree of dissociation $\alpha$ are considered together with the MAL equation (5). For the contact value $h_{\mathrm{D}}(r)$ needed for the calculation $\alpha$ from equation (22) we have

$$
h_{\mathrm{d}}^{00}(R)=-\frac{q_{1}^{00}}{2 \pi \rho R}=-b\left(1+J_{0} R\right)^{2}=-\frac{b}{\left(1+\Gamma_{\mathrm{B}} R\right)^{2}}
$$

and using the exponential form (17) for $g_{+_{-}}(R)$, the equation (5) can be written as

$$
\frac{1-\alpha}{\alpha^{2}}=c 4 \pi N_{\mathrm{A}} B R^{2}\left[1+h_{\mathrm{s}}^{00}(R)\right] \exp \left[\frac{b}{\left(1+\Gamma_{\mathrm{B}} R\right)^{2}}\right] .
$$

In the AMSA the contact value of $1+h_{\mathrm{s}}^{00}(R)$ is equal to the Percus-Yevick value for the hard-sphere model $[5,9]$

$$
1+h_{\mathrm{s}}^{00}(R)=\frac{1+0.5 \eta}{(1-\eta)^{2}}
$$

where $\eta=\frac{1}{6} \pi \rho R^{3}$.

The analysis of equation (30) for $J_{0}$ or equation (32) for $\Gamma_{\mathrm{B}}$ suggests the existence of two different regimes, namely the weak $(\alpha \rightarrow 1)$ and the strong $(\alpha \rightarrow 0)$ 
association regimes. In the regime of weak association equation (32) for $\Gamma_{\mathrm{B}}$ reduces to

$$
4\left(\Gamma_{\mathrm{B}} R\right)^{2}\left(1+\Gamma_{\mathrm{B}} R\right)^{2}=(\kappa R)^{2} \alpha
$$

and in this regime only the electrostatic contribution of free ions is important; contributions from ion pairs can be neglected. The regime of weak association corresponds to the traditional description of electrostatic ion association [7-9] and is realized for

$$
1 \geqslant \alpha \gg \frac{\Gamma_{\mathrm{B}} R}{\left(1+2 \Gamma_{\mathrm{B}} R\right)} .
$$

In the strong association regime, equation (32) for $\Gamma_{\mathrm{B}}$ reduces to

$$
4 \Gamma_{\mathrm{B}} R\left(1+\Gamma_{\mathrm{B}} R\right)^{3}=(\kappa R)^{2}(1-\alpha)
$$

and only the electrostatic contribution from ion pairs is important; the contribution from free ions can be neglected. This regime is realized for

$$
0 \leqslant \alpha \ll \frac{\Gamma_{\mathrm{B}} R}{1+2 \Gamma_{\mathrm{B}} R}
$$

In the AMSA the thermodynamic properties contain three different contributions: the hard sphere contribution (HS), the contribution from the mass action law (MAL) and a contribution from electrostatic ionic interaction (el.) [10,21,22]. The osmotic coefficient is

$$
\Phi=\frac{p_{\mathrm{osm}}}{\rho k T}=\frac{p^{\mathrm{HS}}}{\rho k T}+\frac{p^{\mathrm{MAL}}}{\rho k T}+\frac{p^{\mathrm{el}}}{\rho k T},
$$

where the hard sphere contribution may be calculated by the Carnahan-Starling expression for hard spheres [23]

$$
\frac{P^{\mathrm{HS}}}{\rho k T}=\frac{1+\eta+\eta^{2}-\eta^{3}}{(1-\eta)^{3}}
$$

The effect of ion pair formation on the hard sphere contributions can be calculated with the help of thermodynamic perturbation theory [20] and is included into the MAL-terms

$$
\frac{p^{\mathrm{MAL}}}{\rho k T}=-\frac{1}{2}(1-\alpha)\left[1+\rho \frac{\partial \ln h_{\mathrm{s}}(R)}{\partial \rho}\right]=-\frac{1}{2}(1-\alpha) \frac{1+\eta-0.5 \eta^{2}}{(1-\eta)(1-0.5 \eta)}
$$

The electrostatic contribution can be obtained by the direct integration of the internal energy as shown by Bernard and Blum [21]

$$
\frac{p^{\mathrm{el}}}{\rho k T}=-\frac{\left(\Gamma_{\mathrm{B}} R\right)^{3}}{3 \pi \rho R^{3}}
$$

For the activity coefficient of the free ions one has

$$
\ln y_{ \pm}=\ln y_{ \pm}^{\mathrm{HS}}+\ln y_{ \pm}^{\mathrm{MAL}}+\ln y_{ \pm}^{\mathrm{el}}
$$


where in the same approximation as for osmotic coefficient

$$
\begin{aligned}
\ln y_{ \pm}^{\mathrm{HS}} & =\frac{(1+2 \eta)^{2}}{(1-\eta)^{4}} \\
\ln y_{ \pm}^{\mathrm{MAL}} & =\ln \alpha-\frac{1}{4}(1-\alpha) \rho \frac{\partial \ln h_{\mathrm{s}}(R)}{\partial \rho}=\ln \alpha-\frac{1}{4}(1-\alpha) \frac{5 \eta-2 \eta^{2}}{(1-\eta)(1-0.5 \eta)} \\
\ln y_{ \pm}^{\mathrm{el}} & =-b \frac{\Gamma_{\mathrm{B}} R}{1+\Gamma_{\mathrm{B}} R} .
\end{aligned}
$$

From the comparison of equations (5), (6), (34) and (47) the electrostatic part of the activity coefficient of ionic pairs is obtained

$$
\ln y_{0}^{\mathrm{el}}=-b \frac{\left(\Gamma_{\mathrm{B}} R\right)^{2}}{\left(1+\Gamma_{\mathrm{B}} R\right)^{2}} .
$$

\subsection{Modified AMSA including the effect of ion trimers and tetramers}

The formation of ion aggregates which are more complex than ion pairs was first introduced to explain the conductivity of low permittivity electrolyte solutions [24]. Later on it was shown that this concept is also important for the explanation of other phenomena [9]. We suppose that in the modified AMSA the formation of trimers and tetramers follows the reaction

$$
\left(C^{+} A^{-}\right)^{0}+C^{+}\left(A^{-}\right) \leftrightarrow\left[\left(C^{+} A^{-}\right)^{0} C^{+}\left(A^{-}\right)\right]
$$

where the notation $C^{+}\left(A^{-}\right)$means that the cation $C^{+}$can either be a free ion or is bound in another pair. In the first case we have the formation of ion trimers and in second case we have the formation of tetramers. A relation corresponding to (49) holds for the anion $A^{-}$. As a simplification we consider bilateral triplet formation [9], assuming that the formation of a trimer is characterized by only one association constant $K_{2}$. This means that the concentration of negative trimers $(A C A)^{-}$equals that of positive trimers $(C A C)^{+}$.

Introducing $\gamma c$ as the concentration of ions of a species which is not bound either in a trimer or in a tetramer. Correspondingly $(1-\gamma) c$ will be the concentration of ions of each species bound in trimers and tetramers. Then the ionic solute is assumed to exist in the form of single ions of concentration $\alpha \gamma c$, pairs, $(1-\alpha) \gamma c$, trimers, $\alpha(1-\gamma) c$, and tetramers, $(1-\alpha)(1-\gamma) c$

$$
\alpha \gamma c+(1-\alpha) \gamma c=\gamma c, \quad \alpha(1-\gamma) c+(1-\alpha)(1-\gamma) c=(1-\gamma) c .
$$

Correspondingly, $\alpha c$ is the solute concentration of the charged particles (monomers and trimers) and $(1-\alpha) c$ is the solute concentration of the noncharged particles (pairs and tetramers)

$$
\alpha \gamma c+\alpha(1-\gamma) c=\alpha c, \quad(1-\alpha) \gamma c+(1-\alpha)(1-\gamma) c=(1-\alpha) c
$$


Note that $\alpha c$ is only the solute concentration. The concentration of charged particles of one kind $(+$ or -$)$ is

$$
\alpha \gamma c+\frac{1}{3} \alpha(1-\gamma) c=\frac{2 \gamma+1}{3} \alpha c
$$

For the concentration of the noncharged particles one has

$$
(1-\alpha) \gamma c+\frac{1}{2}(1-\alpha)(1-\gamma) c=\frac{\gamma+1}{2}(1-\alpha) c .
$$

Now we can write the MAL of the ion-pair formation as

$$
\frac{1-\alpha}{\alpha^{2}}=c \gamma K_{\mathrm{A}}\left(1+h_{\mathrm{s}}^{00}(R)\right) \frac{\left(y_{ \pm}^{\mathrm{el}}\right)^{2}}{y_{0}^{\mathrm{el}}}
$$

which differs from equation (2) by the factor $\gamma$. As before $K_{\mathrm{A}}$ is the association constant of ion pairs, for which we can use equation (7). The hard sphere contribution $1+h_{\mathrm{s}}^{00}(R)$ is given by equation $(35)$.

We use the expressions (47) and (49) for the electrostatic contributions of the activity coefficients of free ions, $y_{ \pm}^{\prime}$, and of the ion pairs, $y_{0}^{\prime}$, with correction of equation (32) for $\Gamma_{\mathrm{B}}$. For this purpose, we can neglect the electrostatic contribution from ion trimers and tetramers in $y_{ \pm}^{\mathrm{el}}$ and $y_{0}^{\mathrm{el}}$ as for equation (36) in the regime of weak association, $\alpha \rightarrow 1$. For $\gamma \rightarrow 1$ we generalize the equation for $\Gamma_{\mathrm{B}}$ in the following way

$$
4\left(\Gamma_{\mathrm{B}} R\right)^{2}\left(1+\Gamma_{\mathrm{B}} R\right)^{2}=\gamma(\kappa R)^{2} \frac{\alpha+\Gamma_{\mathrm{B}} R}{1+\Gamma_{\mathrm{B}} R} .
$$

The second MAL relation for the ion trimer and tetramer formation can be written in two different forms. For the regime of weak ion pairing $(\alpha \rightarrow 1)$ the trimer formation process is the dominating process and

$$
\frac{1-\gamma}{\gamma^{2}}=(1-\alpha) y_{2}^{\mathrm{HS}} c K_{2} \frac{y_{ \pm}^{\mathrm{el}} y_{0}^{\mathrm{el}}}{y_{3}^{\mathrm{el}}}
$$

For the regime of strong ionic pairing $(\alpha \rightarrow 0)$ the tetramer formation process is the dominating process and

$$
\frac{1-\gamma}{\gamma^{2}}=(1-\alpha) c y_{2}^{\mathrm{HS}} K_{2} \frac{\left(y_{0}^{\mathrm{el}}\right)^{2}}{y_{4}^{\mathrm{el}}} .
$$

The association constant $K_{2}$ is related to the formation of ion trimers and tetramers and by analogy with the Ebeling association constant it can be given as part of the third ionic virial coefficient in the case of equation (56) or as part of the fourth ionic virial coefficient in the case of equation (57). Third and fourth ionic virial coefficients can be approximated as electrostatic parts of the second virial coefficient for ion-dipole and dipole-dipole interactions, correspondingly [17]. For the case of simplicity we consider $K_{2}$ as an adjustable constant and neglect the hard 
sphere contribution of equations (56)-(57) assuming that $y_{2}^{\mathrm{HS}}=1$. For the electrostatic part of the activity coefficient of the ion trimers, $y_{3}^{\mathrm{e}}$, and ion tetramers, $y_{4}^{\mathrm{e}}$, we assume that by analogy with the relations (47) and (49) we can write

$$
\ln y_{3}^{\mathrm{el}}=-b \frac{\left(\Gamma_{\mathrm{B}} R\right)^{3}}{\left(1+\Gamma_{\mathrm{B}} R\right)^{3}}, \quad \ln y_{4}^{\mathrm{el}}=-b \frac{\left(\Gamma_{\mathrm{B}} R\right)^{4}}{\left(1+\Gamma_{\mathrm{B}} R\right)^{4}} .
$$

The formation of trimers and tetramers is a process following the ion-pair formation. Usually $K_{\mathrm{A}} \gg K_{2}$ [9]. This leads to the following order of the quantities $\alpha$ and $\gamma$

$$
0 \leqslant \alpha \leqslant \gamma \leqslant 1
$$

Due to this fact equation (57) is preferable.

The equations (54), (55) and (57) for $\alpha, \gamma$ and $\left(\Gamma_{\mathrm{B}} R\right)$ form a nonlinear system which in the general case can be solved numerically, e.g. by a Newton-Raphson technique.

After including trimers and tetramers the osmotic coefficient $\Phi$ has a form similar to equation (40), where the hard sphere contribution $P_{\mathrm{HS}} / \rho k T$ is again given by equation (41). For the MAL-contribution we take into account only the ideal terms from free ions, ion pairs, trimers and tetramers. As a result instead of equation (42) we have

$$
\frac{P^{\mathrm{MAL}}}{\rho k T}=\alpha \gamma+\frac{1}{2}(1-\alpha) \gamma+\frac{1}{3} \alpha(1-\gamma)+\frac{1}{4}(1-\alpha)(1-\gamma)-1 .
$$

The electrostatic excess contribution is given by equation (43) where $\Gamma_{\mathrm{B}}$ is calculated from equation (55). We note that due to the approximation made for the calculation of $\Gamma_{\mathrm{B}}$ in equation (55) we neglect in this calculation electrostatic contributions from trimers and tetramers for the osmotic coefficient because with $\gamma \rightarrow 0$ also $\Gamma_{\mathrm{B}} \rightarrow 0$.

The activity coefficient of the free ions has the form (44) with a similar modification for $y_{ \pm}^{\mathrm{MAL}}$ and $y_{ \pm}^{\mathrm{el}}$.

The results obtained are also useful for the calculation of the ionic conductivity of nonaqueous electrolyte solutions. Several attempts exist for the calculation of the molar conductivity of associating electrolytes beyond the limiting law in the chemical model at the level of the mean spherical approximation $[9,25,26]$, where, however, only ion pairs were taken into account. Ion pairs and tetramers are electrically neutral, non-conducting species in the solution, by contrast to the ion trimers. The total concentration of charged particles is given by equation (52) and due to this we modify the result obtained by Turq et al. [25,26], changing $\alpha$ to $\frac{1}{3} \alpha(2 \gamma+1)$ and $\Gamma$ to $\Gamma_{\mathrm{B}}$. Then we have the following expression for molar conductivity

$$
\Lambda=\alpha \frac{(2 \gamma+1)}{3} \Lambda_{0}\left[\left[1+S^{\mathrm{rel}}\left(\alpha \frac{2 \gamma+1}{3}\right)\right]\left[1+S^{\mathrm{el}}\left(\alpha \frac{2 \gamma+1}{3}\right)\right]+\frac{3}{2}\left[S^{\mathrm{rel}}\left(\alpha \frac{2 \gamma+1}{3}\right)\right]^{2}\right]
$$

where $\Lambda_{0}$ is the limiting molar conductivity of the salt in the form of simple ions at infinite dilution. 
The first order relaxation contribution $S^{\text {rel }}$ and the first order electrophoretic term $S^{\mathrm{el}}$ are given by

$$
\begin{aligned}
S^{\text {rel }} & =-\frac{b}{6} \frac{1-\exp \left(-\sqrt{2} \kappa^{\prime} R\right)}{\left(1+\Gamma_{\mathrm{B}} R\right)^{2}+\sqrt{2}\left(1+\Gamma_{\mathrm{B}} R\right)+1-\exp \left(\kappa^{\prime} R / \sqrt{2}\right)}, \\
S^{\mathrm{el}} & =-\frac{N_{a} e^{2}}{3 \pi \eta_{0} \Lambda_{0}} \frac{2 \Gamma_{\mathrm{B}}}{1+\Gamma_{\mathrm{B}} R},
\end{aligned}
$$

where $\left(\kappa^{\prime}\right)^{2}=\kappa^{2} \alpha \frac{2 \gamma+1}{3}$ is the Debye-Huckel screening parameter for charged particles, $\eta_{0}$ is the solvent viscosity.

\section{Experimental data}

Ionic association occurring as a result of strong Coulomb interactions increases at decreasing solvent permittivity $\varepsilon$. However, ionic association also occurs as a result of chemical interactions due to specific solvation forces [9]. In the following discussions on solutions of $\mathrm{LiClO}_{4}$ we focus our attention only on the first case. $\mathrm{LiClO}_{4}$ is known for its remarkable solubility in low permittivity solvents.

To compare theoretical and experimental data, experimental osmotic coefficients were chosen for acetonitrile and aceton [27], 2-propanol [28], 1,2 dimethoxyethane (DME) and dimethylcarbonate (DMC) [29] solutions of $\mathrm{LiClO}_{4}$ with solvents of relative permittivities $\varepsilon=35.95$ for acetonitrile, $\varepsilon=20.70$ for acetone, $\varepsilon=19.39$ for 2-propanol, covering the range $20<K_{\mathrm{A}}\left[\mathrm{mol}^{-1} \mathrm{dm}^{3}\right]<2000$ of the association constant. For electrolytes with solvents of significantly lower permittivities, $\varepsilon=7.02$ for DME and $\varepsilon=3.09$ for DMC, the association constant is much higher $\left(K_{\mathrm{A}}>\right.$ $\left.10^{6} \mathrm{~mol}^{-1} \mathrm{dm}^{3}\right)$.

Osmotic coefficients $\Phi^{\mathrm{LR}}$ were obtained by measurements of vapour pressures $p$ at molalities $m$ and temperature $T$ from vapour pressure lowering $\Delta p=p^{*}-p$, with $p^{*}$ - the vapour pressure of the pure solvent, using the relationship

$$
\Phi^{\mathrm{LR}}=-\frac{1}{2 m M_{\mathrm{s}}}\left[\ln \frac{p^{*}-\Delta p}{p^{*}}-\frac{\Delta p\left(B-V_{\mathrm{s}}^{*(l)}\right.}{R T}\right],
$$

where $M_{\mathrm{s}}$ is the molar mass of the solvent, $B$ is the second virial coefficient of the gas phase solvent, $V_{\mathrm{s}}^{*(l)}$ is the molar volume of the liquid solvent, $R=k N_{\mathrm{A}}$ is the gas constant.

To compare with theoretical data, the experimental osmotic coefficients in the Lewis-Randall (LR) system must be converted to the McMillan-Mayer (MM) system and molarity $c$

$$
\Phi=\Phi^{\mathrm{LR}}\left(1+10^{-3} m M_{\mathrm{E}}\right) \frac{d^{*}}{d}, \quad \Phi_{\mathrm{c}}=\frac{m d}{\left(1+10^{-3} m M_{\mathrm{E}}\right)},
$$

where $d$ and $d^{*}$ are the densities of solution and solvent, $M_{\mathrm{E}}=105.39 \mathrm{~g} / \mathrm{mol}$ is the molar mass of salt $\mathrm{LiClO}_{4}$. The densities are available in the form of polynomials

$$
d=d^{*}+\sum_{i=1}^{3} A_{i} m^{i}
$$


Table 1. Solvent properties.

\begin{tabular}{|c|c|c|c|c|c|}
\hline Solvent & acetonitrile & acetone & 2-propanol & DME & DMC \\
\hline$M_{\mathrm{s}}[\mathrm{g} / \mathrm{mol}]$ & 41.053 & 58.08 & 60.069 & 90.123 & 90.079 \\
\hline$B\left[\mathrm{~cm}^{3} / \mathrm{mol}\right]$ & -6190 & -2132 & -3424 & -2000 & -1950 \\
\hline$d^{*}\left[\mathrm{~g} / \mathrm{cm}^{3}\right]$ & 0.77675 & 0.78421 & 0.780716 & 0.86135 & 1.06335 \\
\hline$V_{\mathrm{s}}^{*(l)}\left[\mathrm{cm}^{3} / \mathrm{mol}\right]$ & 52.852 & 70.995 & 76.971 & 104.63 & 84.664 \\
\hline$P_{i}[\mathrm{~Pa}]$ & 11745 & 30803 & 5777 & 9136 & 7226 \\
\hline$\varepsilon$ & 35.95 & 20.70 & 19.39 & 7.08 & 3.108 \\
\hline$\eta_{0}[\mathrm{cP}]$ & 0.34 & 0.303 & 2.08 & 0.41 & 0.5902 \\
\hline
\end{tabular}

Table 2. Coefficients of the density polynomials, equation (66) for the various electrolyte solutions of $\mathrm{LiClO}_{4}$.

\begin{tabular}{|c|c|c|c|c|c|}
\hline Solvent & acetone & acetonitrile & 2-propanol & DME & DMC \\
\hline$A_{1} * 10^{2}$ & 8.2131 & 7.1513 & 7.0936 & 7.5609 & 8.087 \\
\hline$A_{2} * 10^{2}$ & -1.5952 & -0.64268 & -0.8510 & 5.3787 & -0.4516 \\
\hline$A_{3} * 10^{3}$ & 10.844 & 0.8780 & 1.972 & -0.4516 & -0.0958 \\
\hline
\end{tabular}

The data used to calculate the osmotic and the activity coefficients for the solutions studied in this paper are taken from [27-29] and are given in table 1 and table 2.

The experimental $\Delta p$ data used to calculate the osmotic coefficients are represented in the cited literature by polynomials of the type

$$
\Delta p=\sum_{i=1}^{5} B_{i} m^{i-1} .
$$

The coefficients $B_{i}$ are summarized in table 3 .

The experimental data for the ionic conductivity of $\mathrm{LiClO}_{4}$ solution in DME and DMC were taken from the references [30] and [31].

Table 3. Vapour pressure lowering $\Delta p=p^{*}-p$ of various electrolyte solutions of $\mathrm{LiClO}_{4}$.

\begin{tabular}{|c|c|c|c|c|c|c|}
\hline Solvent & Conc. range & $B_{1}$ & $B_{2}$ & $B_{3}$ & $B_{4}$ & $B_{5}$ \\
\hline Acetone & $0.02-0.58$ & 0.030530 & 17.7091 & -12.3816 & 22.8147 & -14.5306 \\
\hline Acetonitrile & $0.06-1.15$ & 0.027440 & 5.67914 & -1.43816 & 1.36384 & -0.433865 \\
\hline 2-propanol & $0.05-1.48$ & 0.015177 & 2.74737 & -1.01672 & 1.24942 & -0.232199 \\
\hline DME & $0.02-0.35$ & 0.003686 & 6.57103 & -19.2202 & 47.1899 & -49.6216 \\
\hline DMC & $0.03-1.77$ & 0.052101 & 3.50040 & -4.02156 & 3.40816 & -0.682103 \\
\hline
\end{tabular}




\section{Description of the experimental data with the AMSA}

As shown previously [10] the regime of weak ion pairing with $\Gamma_{\mathrm{B}}$ calculated from equation (36) correctly describes ionic solutions with electrostatic ion association for solvents of dielectric permittivity $\varepsilon>36$. For electrolyte solutions with solvent permittivities in the range $20<\varepsilon<36$ the correct description is obtained in the framework of the usual AMSA theory. This result is illustrated in figure 1, where the osmotic coefficients of $\mathrm{LiClO}_{4}$ in different solvents, derived from vapour pressure measurements, are compared with the predictions of the AMSA. For the association constant, equation (7) is used yielding $K_{\mathrm{A}}$ which is connected with the association parameter $B$ according to equation (6). $\Gamma_{\mathrm{B}}$ and $\alpha$ are calculated from equations (32) and (34). The osmotic and activity coefficients are calculated using equations (40)(43) and (44)-(47) respectively. Figure 1 shows the satisfactory agreement of oneparameter AMSA calculations with the experimental results. Distance parameters between $0.42 \mathrm{~nm}$ and $0.48 \mathrm{~nm}$ in protic solvents are reasonable, they are larger than the sum of ionic radii, indicating contributions of contact and solvent shared ion pairs.

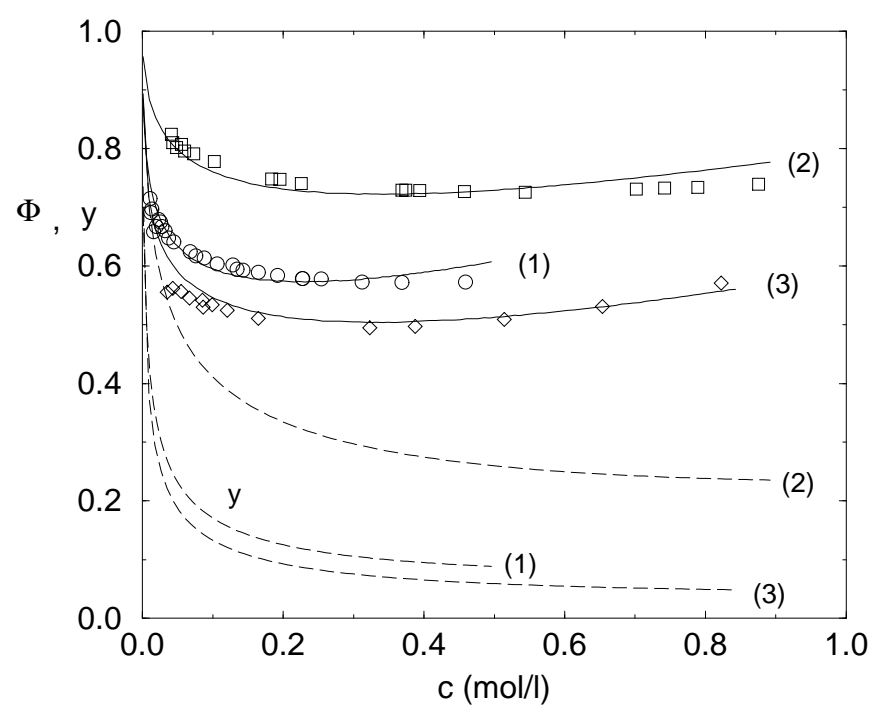

Figure 1. Osmotic coefficients $\Phi$ and mean ionic activity coefficients $y_{ \pm}$of $\mathrm{LiClO}_{4}$ solutions of different solvents at $25^{\circ}$ : Experimental $\Phi$-values: (circles: acetone; squares: acetonitrile; diamonds: 2-propanol). Calculations from AMSA theory (solid lines) (1): acetone, $R=0.48 \mathrm{~nm}$; (2): acetonitrile, $R=0.42 \mathrm{~nm}$; (3): 2-propanol, $R=0.43 \mathrm{~nm}$. Mean ionic activity coefficients: (broken lines).

For ionic solutions of very low permittivity, here for DME and DMC solutions of $\mathrm{LiClO}_{4}$, the occurrence of ion trimers and tetramers should be considered as it is known from conductivity measurements [30,31]. For the description of such ionic solutions we take into account that the permittivity of $\mathrm{LiClO}_{4}$ solutions increases with ionic concentration in both solvents. This effect can be described up to $c=$ $0.3 \mathrm{~mol} / \mathrm{dm}^{3}$ by quadratic polynomials in $c[9]$

$$
\varepsilon=7.08+24 c-21.3 c^{2}
$$


for DME and

$$
\varepsilon=3.11+13.3 c-7.7 c^{2}
$$

for DMC.

Experimental data for the osmotic coefficient of $\mathrm{LiClO}_{4}$ solutions in DMC is known up to $1.77 \mathrm{~mol} / \mathrm{dm}^{3}$ (see table 3 ). For higher concentrations the relation (69) has been altered to a kind of Pade-approximant, to include the saturation effects

$$
\varepsilon=3.11+13.3 c\left(1+\frac{7.7 c}{13.3}\right)^{-1}
$$

The increase of $\varepsilon$ with ionic concentration produces an increase of $\alpha$. This is important to understand the concentration dependence of the electrolyte conductivity. For the calculations, the association constant $K_{\mathrm{A}}$ of equation (7) was used. $\Gamma_{\mathrm{B}}, \alpha$ and $\gamma$ were calculated from equations (54), (55) and (57). The osmotic coefficient is calculated from equations (40), (41), (43) and (60). To calculate the molar conductivity we use equations (61)-(63) with $\Lambda_{0}=176.4 \mathrm{~cm}^{2} \Omega^{-1} \mathrm{~mol}^{-1}$ for $\mathrm{LiClO}_{4}$ solution in DME [30] and $\Lambda_{0}=115.7 \mathrm{~cm}^{2} \Omega^{-1} \mathrm{~mol}^{-1}$ for $\mathrm{LiClO}_{4}$ solution in DMC [31].

Now our approach contains two adjustable parameters, namely the contact distance $R$ and the second association constant $K_{2}$. The calculations show that the osmotic coefficients can be described up to $0.4 \mathrm{~mol} / \mathrm{dm}^{3}$ with fixed $K_{2}=1 \mathrm{dm}^{3} / \mathrm{mol}$ and again with the contact distance as fitting parameter, namely $R=0.318 \mathrm{~nm}$ for $\mathrm{LiClO}_{4}$ solutions in DME and $R=0.1 \mathrm{~nm}$ for $\mathrm{LiClO}_{4}$ solutions in DMC. Distances $R$ up to which two ions are counted as a pair which are much smaller than the ion contact distance, lead to high values of the association constant $K_{\mathrm{A}}$, when calculated from equation (7); $\alpha$ is practically zero and according to equation (61) $\Lambda \approx$ 0 . To avoid this drawback we modify the theory in such a way that we introduce two fitting distances, one for the ionic contact distance, $R$, which we use to calculate the association constant $K_{\mathrm{A}}$ and for the hard-sphere contribution, equation (41), and the other one, $R_{i}$, to calculate the electrostatic contributions connected with the dimensionless screening parameter $\Gamma_{\mathrm{B}} R$, equations $(55)$

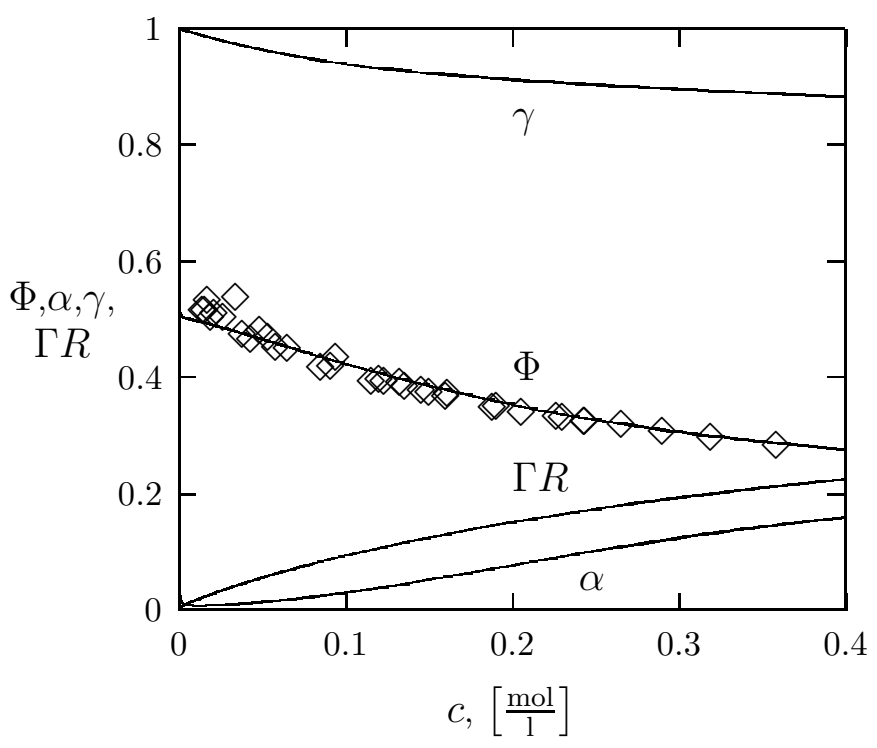

Figure 2. Osmotic coefficients $\Phi$, of $\mathrm{LiClO}_{4}$ solutions in DME. $(\diamond)$ experimental values, (solid line) theoretical values. Also shown: $\gamma$ - the degree of particles not bound in a trimer or a tetramer, and $\alpha$, the degree of charged particles in the system., and the dimensionless screening parameter $\Gamma_{\mathrm{B}} R_{i}$ See the text for details. 
such that a modified $\Gamma_{\mathrm{B}} R_{i}$ term corrects some of the simplifications introduced with the chosen forms of the MAL, equations (55) to (57) and the expressions for the activity coefficients of the higher associates, $y_{3}^{\mathrm{el}}$ and $y_{4}^{\mathrm{el}}$, equation (58).

Figure 2 shows the satisfactory agreement of the modified AMSA with two ionic distances $R=0.42 \mathrm{~nm}$ and $R_{i}=0.22 \mathrm{~nm}$ with the experimental results for the osmotic coefficient of $\mathrm{LiClO}_{4}$ solutions in DME.

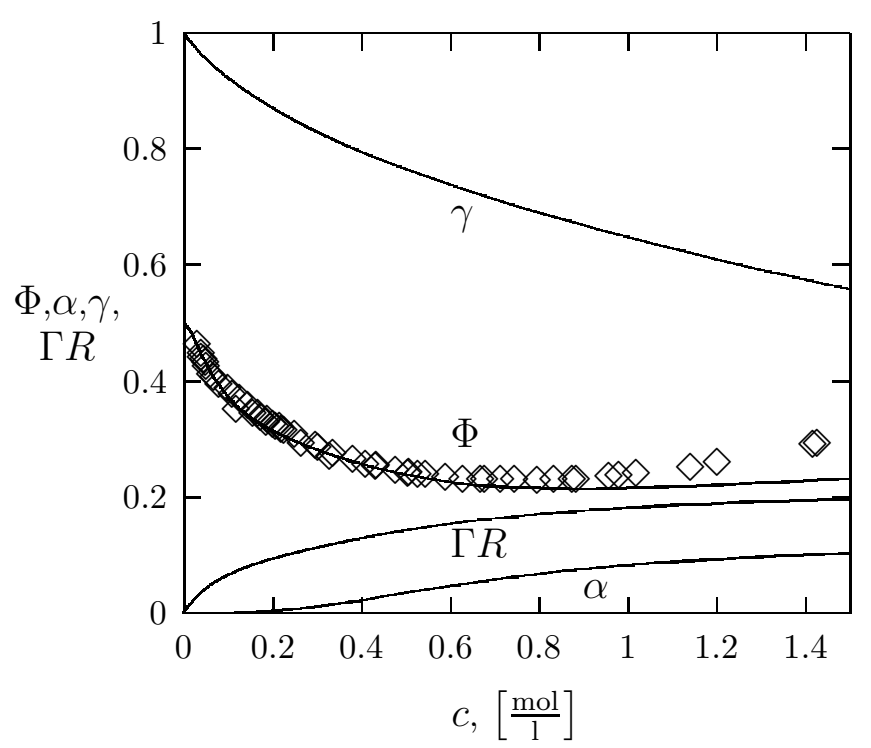

Figure 3. Osmotic coefficients $\Phi$ of $\mathrm{LiClO}_{4}$ solutions in DMC and related quantities (as in figure 2). See the text for details.

Also, the agreement (up to $0.8 \mathrm{~mol} / \mathrm{dm}^{3}$ ) of the modified AMSA theory with $R=$ $0.55 \mathrm{~nm}$ and $R_{i}=0.13 \mathrm{~nm}$ with experimental results of the osmotic coefficient of $\mathrm{LiClO}_{4}$ solutions in DMC is presented in figure 3 . The second association constant for both cases is fixed as $K_{2}=1 \mathrm{dm}^{3} / \mathrm{mol}$.

In figure 2 and figure 3 the concentration dependences of $\alpha, \gamma$ and $\Gamma_{\mathrm{B}} R_{i}$ are also presented. For both cases the $\gamma$ fraction decreases with the ionic concentration in the limit of the applicability of the approximation of equation (55) for $\Gamma_{\mathrm{B}}$. The fraction $\alpha$ strongly decreases, and, beginning from concentrations near $10^{-2} \mathrm{~mol} / \mathrm{dm}^{3}$ slowly increases again. In general $\alpha<0.15$ which justifies the application of the form (57) for the second MAL. Such a behaviour of $\alpha$ and $\gamma$ explains the specific features of the concentration dependence of the conductivity of the considered solutions as shown in the next figures.

A comparison of the experimental data and the theoretical results for the equivalent conductivity obtained from equations (61)-(63) with the parameters used for the description of the osmotic coefficients is given in figure 4 for $\mathrm{LiClO}_{4}$ solutions in DME and in figure 5 for $\mathrm{LiClO}_{4}$ solutions in DMC. In both cases the theoretical curves satisfactorily reproduce the sharp decrease of conductivity for small ionic concentrations due to ion-pair formation, the existence of a conductivity minimum at a concentration near $10^{-2} \mathrm{~mol} / \mathrm{dm}^{3}$ and the increase of conductivity due to ionic trimer formation. For higher concentrations we observe for $\mathrm{LiClO}_{4}$ in $\mathrm{DMC}$ (figure 5) a calculable maximum at a concentration near $1 \mathrm{~mol} / \mathrm{dm}^{3}$ and the decrease of conductivity at an increasing ionic concentration required by tetramer formation. 


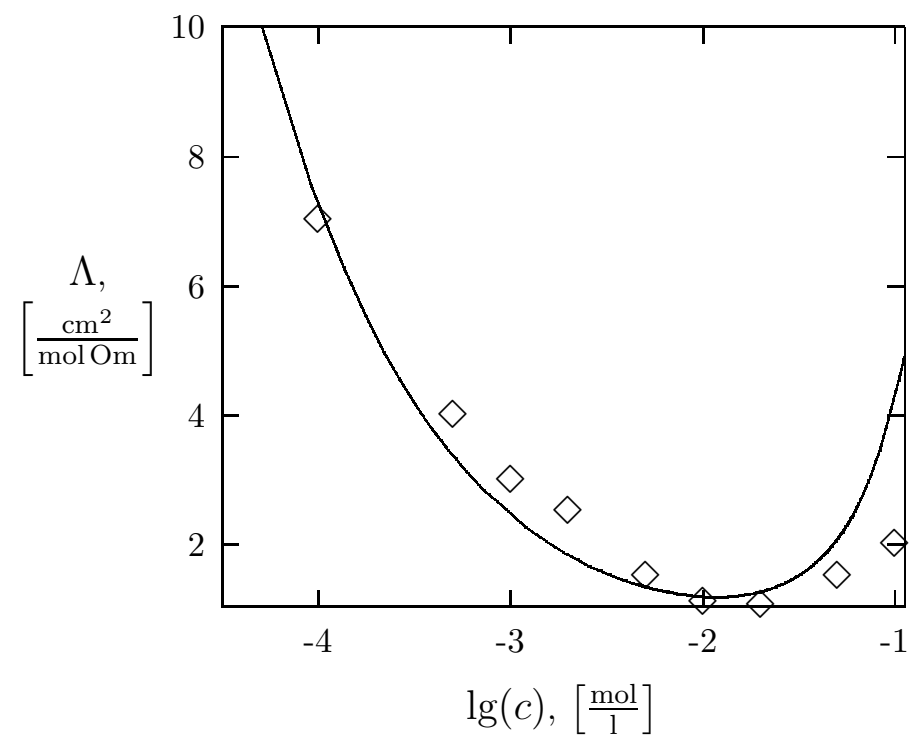

Figure 4. Equivalent conductivity $\Lambda$ of $\mathrm{LiClO}_{4}$ solutions in DME with the parameters used to calculate the osmotic coefficient. See the text for details.

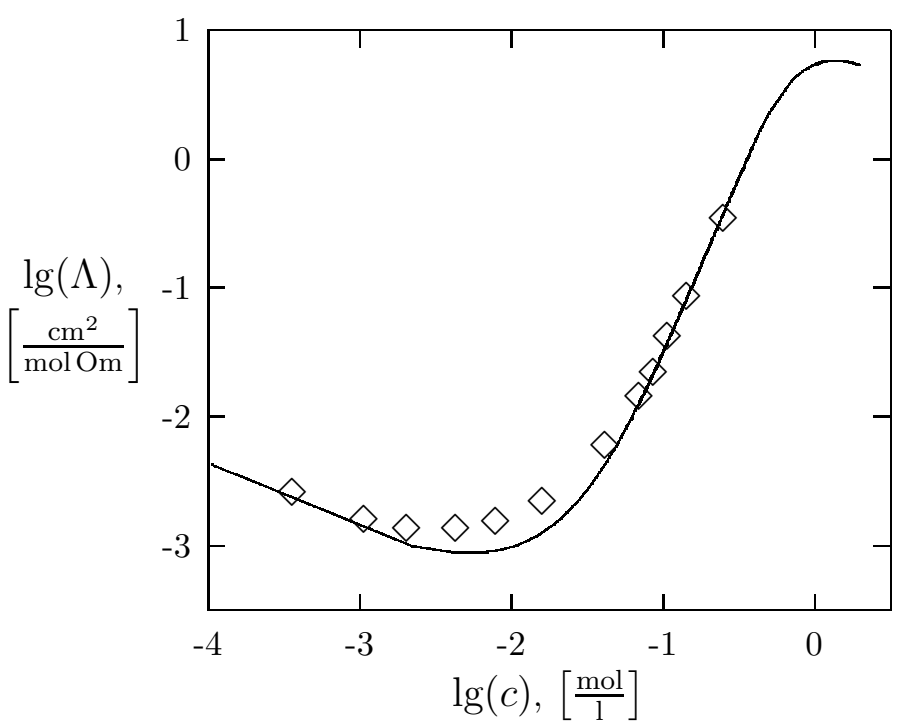

Figure 5. Equivalent conductivity $\Lambda$ of $\mathrm{LiClO}_{4}$ solutions in DMC. See the text for details. 


\section{Conclusions}

An unified approach to the description of the thermodynamic and transport excess functions of associating and nonassociating electrolytes is proposed which leads to numerically simple final formulae capable of presenting experimental data with a minimum of adjustable parameters. These parameters show realistic values in their definition range.

\section{Acknowledgment}

This work was completed in the framework of a cooperation between our institutes at Regensburg and in Lviv, registered under UKR-028-96 at the "Internationales Büro des Bundesministeriums für Bildung, Wissenschaft, Forschung und Technologie (BMBF) bei der Deutschen Luft- und Raumfahrtgesellschaft (DLR)", Germany, and Ministry on Science and Technology of Ukraine. The financial support of this contract is gratefully acknowledged.

\section{References}

1. Yukhnovskij I.R. // Zhurn. Eksp. and Teoret. Fiz., 1954, vol. 27, p. 690 (in Russian).

2. Yukhnovskij I.R. // Zhurn. Eksp. and Teoret. Fiz., 1958, vol. 34, p. 379 (in Russian).

3. Yukhnovskij I.R. // Dokl. Akad. Nauk SSSR, 1961, vol. 136, p. 1317 (in Russian).

4. Yukhnovskij I.R. // Ukr. Fiz. Zhurn., 1962, vol. 7, p. 267 (in Russian).

5. Yukhnovskij I.R., Holovko M.F. The Statistical Theory of the Classical Equilibrium Systems. Naukova dumka, Kiev, 1980.

6. Golovko M.F., Yukhnovskij I.R. - In: "The Chemical Physics of Solvation", Elsevier, Amsterdam, 1985, vol. A, p. 207-262.

7. Bjerrum N. // Mat.-Fys. Medd. K. Dan. Vidensk. Selsk., 1926, vol. 7, p. 1.

8. Barthel J. // Ber. Bunsenges. Phys. Chem., 1979, vol. 83, p. 252.

9. Barthel J., Krienke H., Kunz W. Physical Chemistry of Electrolyte Solutions - Modern Aspects. New York, Steinkopff, Darmstadt and Springer, 1998.

10. Krienke H., Barthel J., Holovko M., Protsykevich I., Kalyuzhnyi Yu. // J. Mol. Liquids, 2000 (in press).

11. Holovko M.F., Kalyuzhnyi Yu.V. // Mol. Phys., 1991, vol. 73, p. 1145.

12. Wertheim M.S. // J. Stat. Phys., 1984, vol. 35, p. 19; p. 35.

13. Wertheim M.S. // J. Stat. Phys., 1986, vol. 42, p. 495; p. 477.

14. Holovko M.F. // Cond. Matt. Phys., 1999, vol. 2(18), p. 205.

15. Ebeling W. // Z. Phys. Chem. (Leipzig), 1968, vol. 238, p. 400.

16. Ebeling W., Grigo M. // Ann. Physik (Leipzig), 1980, vol. 37, p. 21.

17. Holovko M., Protsykevich I., Kapko V., Krienke H., Barthel J. // J. Mol. Liquids, 2000 (in preparation).

18. Waisman E., Lebowitz L. // J. Chem. Phys., 1970, vol. 52, p. 4307.

19. Blum L. // Mol. Phys., 1975, vol. 30, p. 1529.

20. Wertheim M.S. // J. Chem. Phys., 1986, vol. 85, p. 2929.

21. Bernard O., Blum L. // J. Chem. Phys., 1996, vol. 104, p. 4746.

22. Kalyuzhnyi Yu., Holovko M.F. // J. Chem. Phys., 1998, vol. 108, p. 3709. 
23. Carnahan M.F., Starling K.E. // J. Chem. Phys., 1969, vol. 51, p. 635.

24. Fuoss R.M., Kraus C.A. // J. Amer. Chem. Soc., 1933, vol. 55, p. 2387.

25. Chhih A., Turq P., Bernard O., Barthel J., Blum L. // Ber. Bunsenges Phys. Chem., 1994, vol. 98, p. 1516.

26. Turq P., Blum L., Bernard O., Kunz W. // J. Phys. Chem., 1995, vol. 99, p. 822.

27. Barthel J., Neuder R., Poepke H., Wittmann H. // J. Solution Chemistry, 1999 (in press).

28. Barthel J., Neuder R., Poepke H., Wittmann H. // J. Solution Chemitry, 1998, vol. 27, p. 1055.

29. Barthel J., Neuder R., Poepke H., Wittmann H. // J. Solution Chemitry, 1999 (in press).

30. Cachet H., Fekir M., Lestrade J.-C. // Can. J. Chem., 1981, vol. 59, p. 1051.

31. Delsignore M., Farber H., Petrucci S. // J. Phys. Chem., 1985, vol. 89, p. 4968.

\title{
Застосування асоціативного середньосферичного наближення в теорії неводних розчинів електролітів
}

\author{
Й.Бартель ${ }^{1}$, Г.Крінке ${ }^{1}$, М.Ф.Головко ${ }^{2}$, В.І.Капко ${ }^{2}$, \\ І.А.Процикевич ${ }^{2}$ \\ 1 Інститут фізичної і теоретичної хімії, Регенсбурзький університет, \\ D-93040, Регенсбург, Німеччина \\ 2 Інститут фізики конденсованих систем НАН України, \\ 79011 Львів, вул. Свєнціцького, 1
}

Отримано 9 липня 2000 р.

Іонна асоціація в розчинах електролітів досліджується в рамках хімічних моделей. Дано огляд асоціативного середньосферичного наближення (ACCH) в теорії електролітів. Показано, що АССН у комбінації з асоціативною константою Ебелінга задовільно відтворює дані термодинамічних вимірювань, включаючи область високих концентрацій, для неводних розчинів електролітів з розчинниками з діелектричною проникністю в області $20<\varepsilon<36$. Для іонних розчинів 3 низькою діелектричною проникністю АССН модифіковане включенням іонних тримерів і тетрамерів, щоб отримати правильний опис осмотичних коефіцієнтів та іонних провідностей.

Ключові слова: розчини електролітів, асоціативне середньосферичне наближення, осмотичний і активності коефіцієнти, іонна провідність

PACS: $61.20 . \mathrm{Qg}, 66.10 . \mathrm{Ed}$ 